\title{
Correction to: Wage and Hour Law: Guide to Methods and Analysis
}

\author{
Chester Hanvey
}

\section{Correction to:}

\section{Hanvey, Wage and Hour Law, https://doi.org/10.1007/978-3-319-74612-8}

An error in the production process unfortunately led to publication of these chapters prematurely, before incorporation of the final corrections. The version supplied here has been corrected and approved by the author.

\footnotetext{
The updated online version of this book can be found at https://doi.org/10.1007/978-3-319-74612-8 https://doi.org/10.1007/978-3-319-74612-8_1 https://doi.org/10.1007/978-3-319-74612-8_2 https://doi.org/10.1007/978-3-319-74612-8_3 https://doi.org/10.1007/978-3-319-74612-8_4 https://doi.org/10.1007/978-3-319-74612-8_6 https://doi.org/10.1007/978-3-319-74612-8_8 https://doi.org/10.1007/978-3-319-74612-8_9
} 